\title{
Real time Proton Transfer Reaction and Electronic Nose simultaneous measurements on same samples
}

\author{
R. Capuano ${ }^{1}$, I. Khomenko ${ }^{2}$, L. Quercia ${ }^{1}$, A. Catini $^{1}$ \\ R. Paolesse ${ }^{3}$, F. Biasioli ${ }^{2}, \underline{\text { C. Di Natale }}{ }^{1}$ \\ 1. Dept. Electronic Department, University of Rome Tor Vergata, Rome, Italy. \\ 2. Dept. Food Quality and Nutrition, Fondazione E. Mach. S. Michele all'Adige (TN), Italy \\ 3. Dep. Of Chemical Science and Technology-University of Tor Vergata (RM), Italy
}

\begin{abstract}
Electronic noses have been used for fast characterization of diverse species of samples. One of the major drawbacks of electronic noses is the lack of knowledge about the chemical species that are actually detected. On the other hand, mass spectrometers are emerging as fast and sensitive methods for real time analysis of gaseous samples. In this paper, these two analytical approaches are connected together in order to fully characterize samples and to ascertain the origin of the electronic nose performance.
\end{abstract}

Key words: proton transfer reaction, time of flight, electronic nose, multivariate analysis, food.

\section{Introduction}

The analysis of volatile organic compounds (VOCs) is of paramount importance for the characterization of samples for food quality and environmental control to emerging fields such as metabolomics.

Arrays of partially selective gas sensors, also known as electronics noses, have been shown to be suitable for this scope. However, the intrinsic lack of selectivity of sensors makes the interpretation of the results rather difficult. For this reason, electronic noses are usually complemented by GC-MS in order to identify the relevant VOCs for each application. However, being the two instruments based on different methodologies their results are hardly comparable.

Direct injection mass spectrometers are good candidates for the real time analysis of VOC mixtures. Among these techniques, Proton Transfer Reaction - Mass Spectrometer (PTRMS) is one of the most promising in terms of rapidity and sensitivity. Furthermore, the moderate molecular fragmentation results in a simplified interpretation of the results [1].

In this paper, we show that PTR-MS and electronic nose can be connected in series to measure the same samples and PTR data can help in interpreting the electronic nose results.

\section{Experimental}

For the scope, an electronic nose made of 12 porphyrinoids coated Quartz Microbalances [2] has been connected in series to a PTR-TOF-MS from Ionicon (Innsbruck Austria) (see Fig. 1). This is an improved version of the PTR-MS instrument where the usual quadrupole detector is replaced with a time of flight mass analyzer. The electronic nose was designed and fabricated at the University of Rome Tor Vergata.

The hyphenated instruments have been tested in an experiment aimed at the discrimination of three groups of tomato pastes: pure, inoculated with Penicilium spp, and inoculated and treated with a natural antifungal agent. Samples, close in sealed vials, were measured by means of a thermally controlled autosampler.

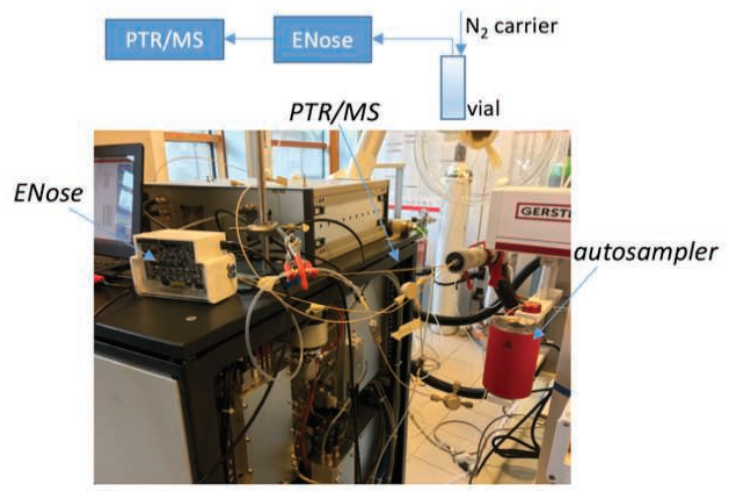

Fig. 1: experimental setup. Both the instruments are connected in series and the sample is carried by constant nitrogen stream kept at $50 \mathrm{sccm}$. 

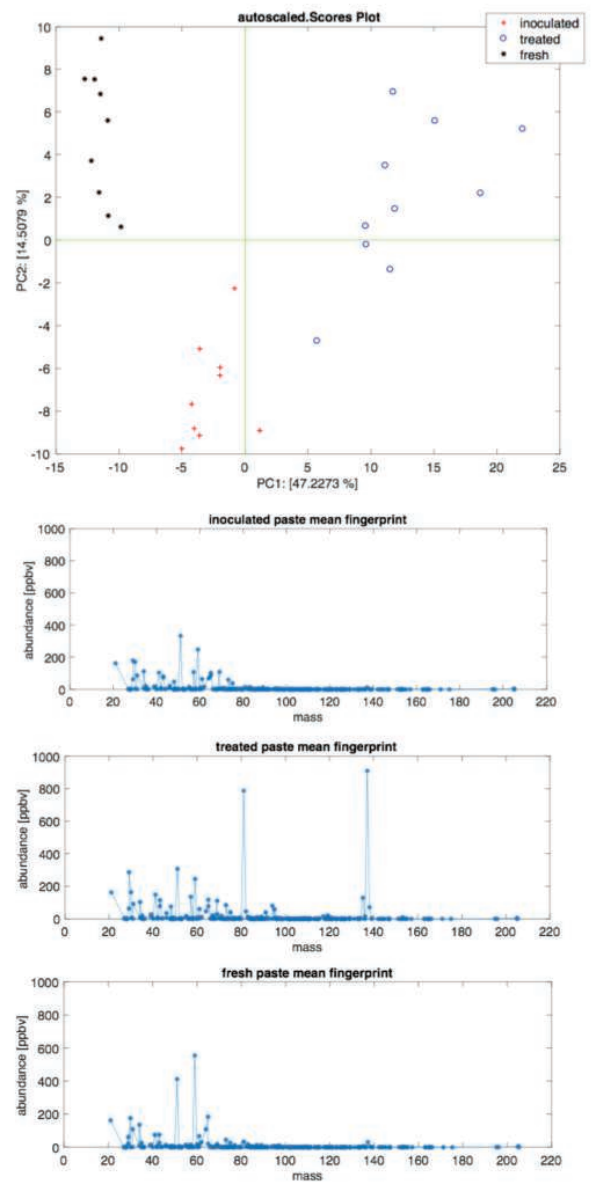

Fig. 2: PCA scores plot of PTR data. For each group of samples the average mass spectra is shown

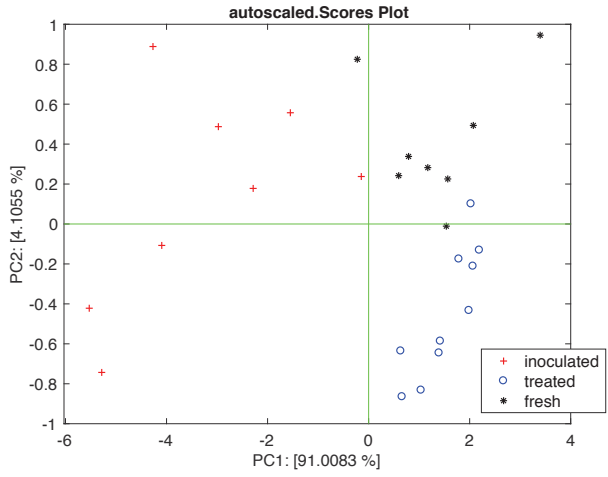

Fig. 3: PCA scores plot of electronic nose data

\section{Results and Discussions}

PTR-MS detected 243 compounds from mass 21 to mass 205. The concentration ranges from sub $\mathrm{ppb}$ to ppm. Fig. 3 shows the principal component analysis (PCA) calculated with the PTR-MS data. For each sample the average mass spectra are also shown. The richest spectra were those related to the pastes treated with antifungal. Fig. 4 shows the PCA of the sensors signal. Here the separation among groups is less sharp but qualitatively similar to
PTR-MS. Differently from PTR-MS, the sensors give the largest signal in case of inoculated pastes. The most interesting use of this methodology is the study of the correlation analysis between the PTR-MS masses and the sensor signals. Fig. 5 shows the linear correlation coefficients between the PTR-MS masses and each of the sensor. The correlation map shows the broad selectivity of the sensors and the subtle differences among sensors. These results aim at removing the serendipitous character of electronic nose providing an optimal tool for accurate and effective design and test of sensors for selected applications. More detailed analysis and the methodology for sensor arrays design will be presented in the final paper.
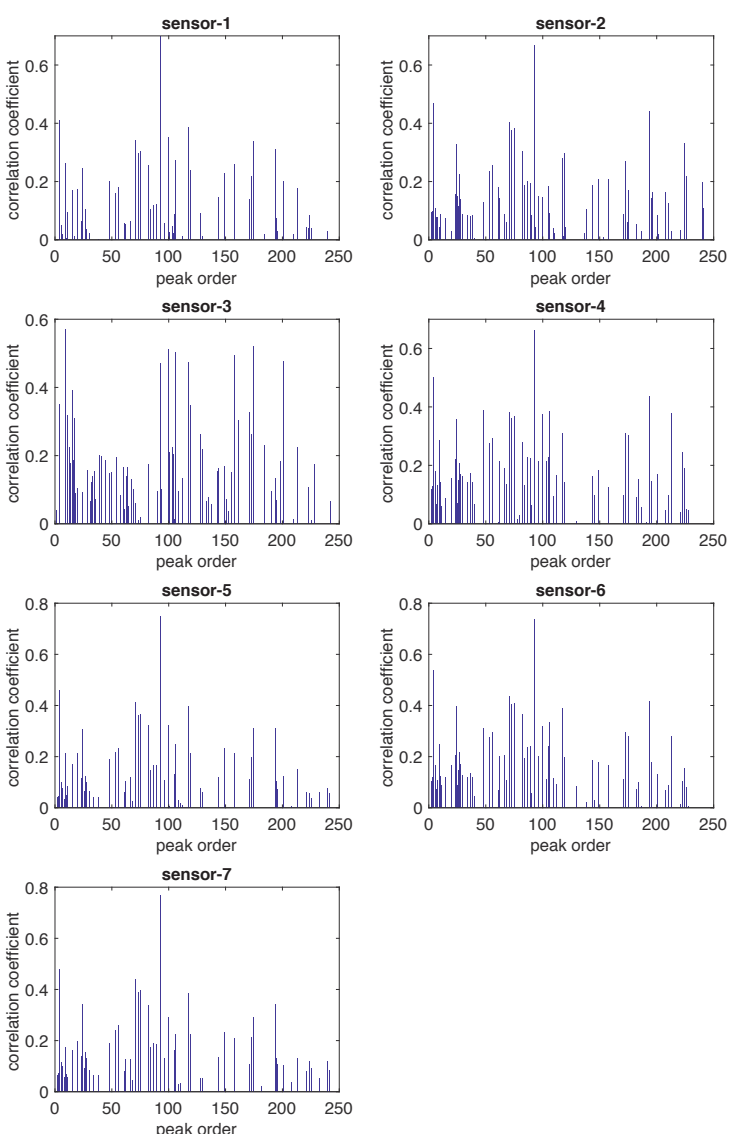

Fig. 4: linear correlation coefficients of mass spectra and 7 sensors of the electronic nose.

\section{References}

[1] F. Biasioli, F. Gasperi, C. Yeretzian, T. Mark. PTR-MS monitoring of VOCs and BVOCs in fodd science Trends in Analytical Chemistry 30, 968977 (2011), 968 doi 10.1016/j.trac.2011.03.009

[2] R. Paolesse, S. Nardis, D. Monti, M. Stefanelli, C. Di Natale, Porphyrinoids for chemical sensors applications. Chem Rev 117, 2517-2583 (2017) doi: 10.1021/acs.chemrev.6b00361 